\title{
The effect of gastrointestinal pathogen panel (GIP) on antibiotic management
}

\author{
Christopher F. Saling MD ${ }^{1}$ (1), Maria T. Seville MD ${ }^{1}$ and Roberto L. Patron MD² \\ ${ }^{1}$ Division of Infectious Diseases, Mayo Clinic, Phoenix, Arizona and ${ }^{2}$ Infectious Disease Providence Medical Group, Portland, Oregon
}

To the Editor-The gastrointestinal pathogen panel (GIP) offers detection via multiplexed polymerase chain reaction (PCR) for a multitude of bacterial, viral, and parasitic microbes associated with diarrheal illness. Its rapid turnaround time and high sensitivity has made GIP testing commonplace for the evaluation of diarrhea. Recent studies demonstrated its utility within the hospital setting. Torres-Miranda et $\mathrm{al}^{1}$ found that GIP testing led to decreased median hospital length of stay (LOS) by 4 days in patients with diarrhea. Furthermore, the mean time to appropriate antibiotic therapy was shortened by 24 hours in those who underwent GIP testing. In a retrospective Dutch study, Machiels et $\mathrm{al}^{2}$ concluded that if GIP had been implemented over conventional cultures, $83 \%$ of patients with diarrhea could have been removed from isolation precautions sooner. Axelrad et $\mathrm{al}^{3}$ conducted a retrospective study involving both inpatients and outpatients with diarrhea and reported that those who underwent GIP testing were less likely to be prescribed antibiotics and to undergo abdominal imaging or endoscopy. The utility of GIP in immunocompromised patients has also been documented. In patients with inflammatory bowel disease (IBD), Hong et $\mathrm{al}^{4}$ showed that GIP testing was associated with increased detection of microbes and lower rates of IBD therapy escalation and endoscopies with no difference in adverse outcomes. GIP testing in hematopoietic stem-cell transplant recipients has been associated with increased identification of infectious pathogens without increasing overall testing cost. ${ }^{5}$

However, despite the aforementioned potential benefits surrounding GIP testing, its high sensitivity to detect pathogens poses significant challenges to proper antibiotic stewardship practices. Also, for most patients, many of the microbes detected via GIP do not warrant antimicrobial treatment, potentially making the test economically wasteful. We examined the impact of GIP on antimicrobial management at our own institution. We conducted a retrospective study of 50 randomly selected patients hospitalized at Mayo Clinic in Arizona who were tested with a BioFire FilmArray GI PCR panel (bioMèrieux, Marcy-l'Étoile, France) between July and December 2019. Medical records were reviewed to capture gender, age, immunocompromised state, antibiotic use within 30 days, prior hospitalization within 3 months, history of Clostridioides difficile infection, time from admission to testing and GIP results. The

Author for correspondence: Christopher F. Saling, MD, Division of Infectious Diseases, Mayo Clinic, 5777 East Mayo Boulevard Phoenix, AZ 85054. E-mail: Christopher.saling@gmail.com

Cite this article: Saling CF, Seville MT, and Patron RL. (2021). The effect of gastrointestinal pathogen panel (GIP) on antibiotic management. Antimicrobial Stewardship \& Healthcare Epidemiology, https://doi.org/10.1017/ash.2021.162 primary endpoint of our study was to determine whether GIP results directly contributed to antibiotic management. This study was exempt from institutional review board approval.

In total, 26 patients were male and 24 were female; the average age was 61.7 years. Among them, 34 patients (68\%) were immunocompromised. Overall, 41 GIP tests were ordered within 24 hours of admission. Of these, 22 patients (44\%) had a positive GIP result and 5 were positive for 2 concurrent organisms. C. difficile was the most detected organism; it was identified in $16(66.7 \%)$ of 24 positive tests. Overall, 11 patients $(68.8 \%)$ with C. difficile had a recent hospitalization, antibiotics within 30 days, or a history of $C$. difficile infection. There were 3 cases of enteropathogenic Escherichia coli, 2 cases of enterotoxigenic Escherichia coli, 2 cases of adenovirus, 2 cases of norovirus, 1 case of rotavirus, and 1 case of Vibrio cholerae. Excluding C. difficile-positive patients, GIP testing changed the antibiotic management in $3(6 \%)$ of 50 patients tested. One patient had antibiotics stopped appropriately; 1 patient received appropriate antibiotics, and 1 patient received inappropriate antimicrobial therapy.

Our results show that, except in the setting of $C$. difficile infection, GIP had little utility in guiding antimicrobial management. This was also true in the immunocompromised patient population. Other studies have similarly shown that $C$. difficile represented most pathogens found on GIP. ${ }^{6}$ Thus, it may be more efficient to first test patients hospitalized with diarrhea for C. difficile alone, especially in those with known risk factors for $C$. difficile infection. Furthermore, GIP testing does not differentiate between $C$. difficile colonization and active disease; therefore, it can lead to overtreatment for C. difficile. Although the GIP panel has many potential advantages compared to traditional testing with culture and ova and parasite examination, interpreting its results can prove difficult. Infectious disease consultants and antimicrobial and diagnostic stewardship teams should be involved to help guide the appropriate use of GIP testing.

\section{Acknowledgments.}

Financial support. No financial support was provided relevant to this article.

Conflicts of interest. All authors report no conflicts of interest relevant to this article.

\section{References}

1. Torres-Miranda D, Karsner R, Secco A, et al. Use of BioFire FilmArray gastrointestinal PCR panel associated with reductions in antibiotic use, time to optimal antibiotics, and length of stay. BMC Gastroenterol 2020; 20:246.

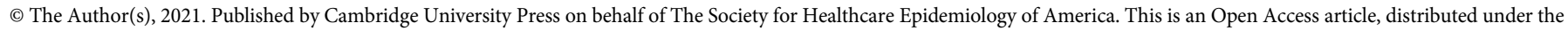
terms of the Creative Commons Attribution licence (http://creativecommons.org/licenses/by/4.0/), which permits unrestricted re-use, distribution, and reproduction in any medium, provided the original work is properly cited. 
2. Machiels J, Cremers A, van Bergen-Verkuyten M, Paardekoper-Strijbosch S, et al. Impact of the BioFire FilmArray gastrointestinal panel on patient care and infection control. PLoS One 2020;15(2):e0228596.

3. Axelrad J, Freedberg D, Whittier S, et al. Impact of gastrointestinal panel implementation on health care utilization and outcomes. J Clin Microbiol 2019;57(3):e01775-18.

4. Hong S, Zaki T, Main M, et al. Comparative evaluation of conventional stool testing and multiplex molecular panel in outpatients with relapse of inflammatory bowel disease. Inflamm Bowel Dis 2021. doi: 10.1093/ibd/izaa336.
5. Rogers W, Westblade L, Soave R, et al. Impact of multiplexed polymerase chain reaction panel on identifying diarrheal pathogens in hematopoietic cell transplant recipients. Clin Infect Dis 2020;71:1693-1700.

6. O'Neal M, Murray H, Dash S, et al. Evaluating appropriateness and diagnostic stewardship opportunities of multiplex polymerase chain reaction gastrointestinal testing within a hospital system. Ther Adv Infect Dis 2020. doi: $10.1177 / 2049936120959561$. 